Western University

Scholarship@Western

Department of Economics Research Reports

Economics Working Papers Archive

1993

\title{
Multi-Player Bargaining Situations: A Decision Theoretic Approach
}

Murali Agastya

Follow this and additional works at: https://ir.lib.uwo.ca/economicsresrpt

Part of the Economics Commons

Citation of this paper:

Agastya, Murali. "Multi-Player Bargaining Situations: A Decision Theoretic Approach." Department of Economics Research Reports, 9307. London, ON: Department of Economics, University of Western Ontario (1993). 


\section{4}

ISSN : 0318-725X

ISBN : 0-7714-1506-0

\section{RESEARCH REPORT 9307}

\section{Mult1-Player Bargalning Situations:}

A Decision Theoret1c Approach

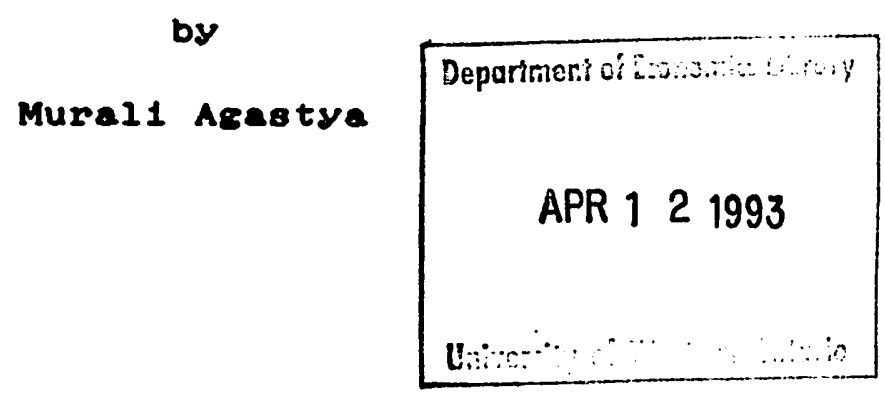

March 1993

Department of Economice

Social Science Centre

UnIversity of Western Ontar1o

London, Ontar1o, Canada

N6A $5 C 2$ 


\title{
Multi-Player Bargaining Situations: A Decision Theoretic Approach
}

\author{
Murali Agastya* \\ Department of Economics \\ University of Western Ontario \\ London, ON N6A 5C2 \\ CANADA
}

March 31, 1993

\begin{abstract}
Often, one has to choose between bargaining situations with a knowledge of the amount of surplus available for sharing but ignorant of the exact process by which this surplus is split. Given the extreme sensitivity of the predictions of the non-cooperative bargaining theory to the specification of the bargaining process, the question arises as to how a typical agent makes this ex-ante choice. In this paper I adopt an axiomatic approach to tackle this problem.

The model also axiomatizes a bargaining solution where the physical outcome is immune to ordinal transformations of the players' preferences for the underlying set of alternatives. The main result is as follows: Assuming that the side-payments can be made in a single infinitely divisible good, the bargaining solution requires that a player be paid a weighted average of his marginal contributions to each coalition. In fact, these weights coincide with the Shapley weights.
\end{abstract}

Keywords: Shapley Value, NTU games, Ordinal Utility, Interpersonal Utility Comparisons, Bargaining Situations

-I thank Phil Reny, Arthur Robson and Abhijit Sengupta for their advice. I also thank Alvin Roth, Herve Moulin and Motty Perry for comments on an earlier draft. The usual disclaimer applies. 


\section{Introduction}

The last decade has seen a flurry of research activity in non-cooperative bargaining theory. A principal insight gained from this literature is the importance of the exact specification of the bargaining process in determining the prediction(s). But often, a typical agent has to choose between multilateral game (or conflict) situations without a complete knowledge of the rules of the game. For example, consider a firm contemplating collaboration in foreign markets or a candidate on the job- market who has to decide which firm to join. If one subscribes to the basic tenet that agents make consistent choices, the question arises as to how they make those choices. This paper provides a theory of ex-ante choice for a class of game situations.

I consider those physical environments where side-payments are possible in a single good. These are referred to as bargaining situations. Loosely speaking, a bargaining situation consists of a set of $n$ players and a non-negative and super-additive function $f$ defined on the class of all coalitions of this set. Here, $f(S)$ is the quantity of a certain infinitely divisible and transferable commodity available to a coalition $S$. Side-payments are made in this good. I postulate a binary relation $\succeq_{i}$ on the class of all bargaining situations. A player anticipates how much of the good he will get should he find himself in any of the bargaining situations. Thus by $f \succeq_{i} g$, we mean that player $i$ expects to obtain at least as much of the good in situation $f$ as in $g$. The strategy of this paper is to impose restrictions on this binary relation. A representation of $\succeq_{i}$ is the utility of a certain bargaining situation for the player in terms of the underlying good itself.

The main result of the paper is as follows. For a bargaining situation $f,[f(S)-f(S \backslash i)]$ is the marginal contribution of player $i$ to the coalition $S$. Under certain intuitive axioms on $\succeq_{i}$, it is shown that in choosing between bargaining situations, an agent maximizes a weighted average of his marginal contributions to all the possible coalitions. Theorem 3 shows that these weights in 
fact coincide with the Shapley weights ${ }^{1}$.

That the weights obtained in Theorem 3 coincide with the Shapley weights may lead one to interpret this representation theorem as only a recharacterization of the Shapley value. Theorem 3 is in fact much more general. The initial construction of the Shapley value is for games with transferable utility. Transferable Utility(TU) requires a single composite good for making sidepayments. Moreover, it is necessary that the utility for this good be linear for all the players, with the same scales. While I also assume that side-payments are possible in a single good, I do not assume linear utility. In fact, the exact utility for the good is irrelevant. The only thing that matters is the implicit assumption that the players prefer more of the good to less of it.

This irrelevance of the exact utility for the underlying good provides us with several insights into bargaining theory and value theory. For instance, Theorem 3 determines a bargaining solution. This bargaining solution is trivially immune to ordinal transformations of the players utility for this good. Thus the model here provides a resolution to the Bargainers Paradox. (See Shapley(1969) or Shubik(1982).) The above bargaining solution also determines a pareto-optimal allocation without actually imposing it as an axiom. Moreover, given that only side- payments are assumed, the model characterizes a non-transferable utility(NTU) value for the above physical environments. And finally, the approach adopted in this paper provides a framework for a proper interpretation of value as the ex-ante evaluation of a game. To ease exposition, an elaborate discussion of various issues raised in this paragraph is deferred to Section 4 . Section 4 also compares the present work to the relevant literature. The model and the axioms are laid out in Section 2. Section 3 contains all the results. Section 5 concludes. All formal proofs are in the Appendix.

\footnotetext{
${ }^{1}$ The literature on Shapley value is enormous. See Roth(1988) for a list of references.
} 


\section{The Model}

Let $N=\{1,2, \ldots n\}$ be a finite set denoting the set of players. A coalition is a non-empty subset of $N$. Given coalitions $S, T, U, \ldots$, let $s, t, u \ldots$ denote the number of players in the respective coalitions. A non-negative function, $f$, defined on the class of all coalitions is said to be superadditive if, for any two disjoint coalitions $S$ and $T, f(S \cup T) \geq f(S)+f(T)$. As is usual, let $2^{N}$ denote the class of all subsets of $N$. Furthermore, let $\Pi_{i}$ denote the class of all coalitions that contain player $i$ and $\Pi_{-i}$ denote the class of all coalitions that do not contain player $i$.

Definition 1 A n-player bargaining situation is a non-negative function $f: 2^{N} \longrightarrow \Re_{+}$such that

\section{1. $f$ is super-additive}

2. $f(\phi)=0$

Let $\mathcal{G}$ denote the class of all bargaining situations. We regard $\mathcal{G}$ as a subset ${ }^{2}$ of $\Re^{2^{N}-1}$. By way of interpretation, given a bargaining situation $f$ and a coalition $S, f(S)$ is the quantity of a single good, say money, that is available to the members of a coalition $S$ if members of $S^{c}$ leave ${ }^{3}$. I assume that side- payments are made in this good. It is worthwhile to re-emphasize that this is not the same as assuming transferable utility.

A typical player $i$, is completely characterized by a complete, transitive and reflexive relation $\succeq_{i}$ defined on $\mathcal{G}$. Such a relation is called a preference relation. Thus, given two bargaining situations $f$ and $g, f \succeq_{i} g$ should be read as "player $i$ weakly prefers the prospect of being in situation $f$ to the prospect of being in situation $g$ ". The symbols $\succ_{i}$ and $\sim_{i}$ have the usual interpretation of strict preference and indifference.

\footnotetext{
${ }^{2}$ Hence the addition of two bargaining situations or multiplication of a bargaining situation by a scalar are well defined.

${ }^{3}$ See Hart and Mas- Colell(1992) for a discussion on this issue.
} 
Certain situations play a key role in the analysis. Let $f_{\phi}$ denote the null situation, that is $f_{\phi}(S)=0$, for all $S \subseteq N$. Formally, this situation corresponds to the origin of $\Re^{2^{N}-1}$ of which $\mathcal{G}$ is a subset. In $f_{\phi}$, no coalition is productive. At the other extreme, there are situations in which the presence of a certain coalition is necessary and sufficient for production. Formally, given a coalition $S$, let $\delta_{S}$ the following situation:

$$
\delta_{S}(T)= \begin{cases}1 & \text { if } S \subseteq T \\ 0 & \text { otherwise }\end{cases}
$$

In the situation $\delta_{S}$, the members of $S$ are solely responsible for any surplus that is available to a coalition $T$. For a $x \geq 0$, I will refer to $x \delta_{S}$ as a pure bargaining situation(PBS) of size $x$ for $S$. A PBS is said to include player $i$ if it is a PBS for a coalition $S$ that contains $i$. A PBS is easy to analyze. Hence the strategy will be to impose intuitive axioms on the $\succeq_{i}$ between various PBS and then extend this on to more complex bargaining situations.

The first axiom is a continuity assumption. It is well known that such an assumption is a minimal requirement to ensure the existence of any real representation.

A 1: (Continuity): The graph of $\succeq_{i}$ is closed, for all $i=1,2, \ldots N$.

Consider $\delta_{S}$ and $x \delta_{S}$ where $x$ is very close to one. Each is a PBS for $S$ and they are approximately of the same size. If a player $i$ who is not in $S$ is asked to choose between the above two situations, it seems reasonable to assume that he will be indifferent between the two. This is the content of the next axiom.

A 2: (Nullity) Suppose $i \notin S$. There exists an $x \neq 1$ such that $\delta_{S} \sim_{i} x \delta_{S}$.

REMARK: A game with TU, say $v$, is said to be null for player $i$ if, for every coalition $S$, $v(S)=v(S \backslash i)$. In a related context, Roth(1988) requires that if two games $v$ and $w$ are null for 
player $i$, then $v \sim_{i} w$. Since $v_{\phi}$, the game analogous to $f_{\phi}$ is null for a player, this assumption basically assumes that in a null situation, a player obtains nothing. Although the implication of the Nullity axiom stated here is the same, i.e. players outside $S$ in a pure-bargaining situation for $S$ evaluate it as if they obtained nothing, I do not assume this outright. Technically, the assumption here is weaker.

Now consider $x \delta_{S}$ and $y \delta_{N \backslash S}$ with $x>y$. Suppose player $i$ is in $S$. The next axiom requires that player $i$ strictly prefer a larger PBS (that is $x \delta_{S}$ ) that includes him to a PBS (that is $y \delta_{N \backslash S}$ ) of smaller size that does not include him.

A 3: (Productivity) Suppose $i \in S$. Then for every $x>y \geq 0, x \delta_{S} \succ_{i} y \delta_{N \backslash S}$.

An alternate interpretation is as follows: Player $i$ has to choose between an organization $S \backslash i$ and an organization $N \backslash S$. Player $i$ 's presence will enable $S \backslash i$ produce $x$ units and nothing otherwise. On joining $N \backslash S$, the production will continue to be $y$ units, which is strictly less than x. Productivity requires that player $i$ choose $S \backslash i$.

Suppose player $i$ prefers $f$ to $g$. Now suppose player $i$ is informed that in both $f$ and $g$ a coalition $S$ is able to produce an extra unit of output. That is, he is now asked to choose between $f+\delta_{S}$ and $g+\delta_{S}$. The next axiom states that he does not change his preference.

A 4: (Strategic Equivalence) Suppose $f \succ_{i} g$. Then for all $x \geq 0, f+x \delta_{s} \succ_{i} g+x \delta_{s}$.

Axioms A5 and A6 below are of a different nature compared to axioms A1-A4. The first four axioms are concerned with the preferences of a single player. In the next two axioms I tie in the preferences of various players. A5 is a simple Symmetry assumption. Such an assumption is often of some interest.

In $\delta_{i}$, one expects player $i$ to obtain the 1 unit. Consider a PBS that includes players $i$ and $j$. 
The Symmetry assumption requires that if the above PBS is large enough for a player $i$ to prefer it to $\delta_{i}$ (and hence player $i$ expects to obtain more than one unit), then the PBS is large enough for player $j$ to at least weakly prefer it to $\delta_{j}$.

A 5: (Symmetry): Suppose $i, j \in S$. For any real number $x$, if $x \delta_{S} \succ_{i} \delta_{i}$, then $x \delta_{S} \succeq_{j} \delta_{j}$

REMARK: In value theory, typically a Symmetry assumption is made(See Shapley(1953)). The Symmetry assumption made here is specific to a particular coalition.

To introduce the next axiom, it is helpful to introduce some definitions. To understand the motivation for the terminology, it is useful to recall that in $\delta_{i}$, one expects player $i$ to keep the entire surplus for himself.

Definition 2 Suppose $i \in S$ and $0 \leq x<|S|$. Player $i$ is said to be an optimist if $x \delta_{S} \succ_{i} \delta_{i}$

Similarly, one defines an pessimist as follows:

Definition 3 Suppose $i \in S$ and $x>|S|$. Player $i$ is said to be a pessimist if $\delta_{i} \succ_{i} x \delta_{S}$.

Suppose there are only two players, say $A$ and $B$. Furthermore, suppose, in a loose and informal sense, that $\left(\succeq_{A}, \succeq_{B}\right)$ are common-knowledge between the two players. That is, both players know the preferences of the other player, both players know that both players knows the preferences of the other player, ... and so on ad infinitum. Now consider a PBS of size $x$ for the coalition $A B$, where $x<2$. Suppose that, at the chosen $x$ both $A$ and $B$ are optimists. So $B$ expects to obtain more than one unit in $x \delta_{A B}$ and so does $A$. Moreover both players know that each of them expects to get more than one unit, and both players know that both players know that both of them expects more than one unit in $x \delta_{A B}$. Since there is not enough surplus to go around, this cannot happen.

Now suppose that $x>2$ and player $B$ is a pessimist. So $B$ expects to obtain less than one unit. Now, both players prefer more of the good to less of it. If $B$ is a pessimist, it must be because 
he expects player $A$ to obtain more than one unit. If fact, given our assumption of commonknowledge, not only does $B$ expect $A$ to obtain more than one unit, he knows that $A$ obtains more than one unit. Hence when $A$ has to choose between $x \delta_{A B}$ and $\delta_{A}$, he will opt for the former. Axiom 6 formalizes this intuition.

A 6: (Consistency) For a PBS of size $x$ for a coalition $S$,

1. If $x<|S|$, at least one player in $S$ is not an optimist.

2. If $x>|S|$, at least one player in $S$ is not a pessimist.

\section{Representation Theorems}

In this section, I state all the Theorems. I provide a sketch of the proof for Theorem 1 . The sketch also highlights the role of the various axioms. All formal arguments are in the appendix.

Theorem 1 Consider a preference relation $\succeq_{i}$ on $\mathcal{G}$. The following are equivalent:

1. The preference relation $\succeq_{i}$ satisfies $A 1-A 4$.

2. There is a unique set of positive weights $\left\{p(i, S): S \in \Pi_{i}\right\}$, such that $\sum_{S \in \Pi_{i}} p(i, S)=1$ and

$$
f \succ_{i} g \Longleftrightarrow \sum_{S \in \Pi_{i}} p(i, S)[f(S)-f(S \backslash i)]>\sum_{S \in \Pi_{i}} p(i, S)[g(S)-g(S \backslash i)]
$$

PROOF:(Sketch) It is easy to see that the second statement implies the first. Suppose now that $\succeq_{i}$ satisfies 2 . We need to show that it satisfies 1 . Towards this end we show that there is an extension $>_{i}^{*}$ of $\succ_{i}$ to the whole of $\Re^{2^{N}-1}$ such that for any three vectors $f, g$, and $h \in \Re^{2^{N}-1}$, $f>_{i}^{*} g$ if and only if $f+h>_{i}^{*} g+h$. This is Lemma 2. In particular, Lemma 2 implies that for any two bargaining situations $f$ and $g, f \succ_{i} g$ if and only if $f-g>_{i}^{*} 0$. Now, let $E_{i}$ be the set of all 
vectors that are strictly better than 0 under $>_{i}^{*}$. Then clearly, $f \succ_{i} g$ if and only if $(f-g) \in E_{i}$. It can be shown(Lemma 5 ) that $E_{i}$ is an open and convex subset of $\Re^{2^{N}-1}$ that does not contain the origin. Hence there is a hyperplane that strictly separates $E_{i}$ and the origin. It is now easy to obtain the following lemma:

Lemma 6 There is a set of weights $\{\lambda(i, S): S \subseteq N\}$ such that for any $f, g \in \mathcal{G}$,

$$
f \succ_{i} g \Longleftrightarrow \sum_{S \subseteq N} \lambda(i, S) f(S)>\sum_{S \subseteq N} \lambda(i, S) g(S)
$$

It may be verified that for any $f$,

$$
\sum_{S \subseteq N} \lambda(i, S) f(S)=\sum_{S \in \Pi_{i}} \lambda(i, S)[f(S)-f(S \backslash i)]+\sum_{S \in \Pi_{-i}}[\lambda(i, S)+\lambda(i, S \cup i)] f(S) .
$$

I use A2 to conclude that $\lambda(i, S)+\lambda(i, S \cup i)=0$, if $S \in \Pi_{-i}$. This is Lemma 7. Finally Lemma 8 shows that $\lambda(i, S)>0$, if $S \in \Pi_{i}$. Now, set $p(i, S)=\frac{\lambda(i, S)}{\sum_{T \in \Pi_{i}}^{\lambda(i, T)}}$.

The uniqueness of the set of weights follows from the fact that the ordering is complete and the weights are normalized to add up to one. This completes the proof.

Armed with Theorem 1, I now proceed to characterize the weights obtained therein more completely by imposing other axioms. Theorem 2 below, states the implication of the Symmetry axiom in addition to those stated in Theorem 1.

Theorem 2 Consider $n$ preference relations $\succ_{i}, i=1,2, \ldots n$, defined on $\mathcal{G}$ such that each of them satisfies A1-A5. Then the weights obtained in Theorem 1 satisfy the following:

$$
\text { If } S \in \Pi_{i} \cap \Pi_{j} \text {, then } p(i, S)=p(j, S) \text {. }
$$

PROOF: See Appendix. 
Note that our assumption of Symmetry is specific to a given coalition. That is, even under the Symmetry axiom, player $i$ 's evaluation of a coalition with player $k$ (and not containing player $j$ ) may be different from player $j$ 's evaluation of a coalition with player $^{4} k$ (and not containing player i). The assumption of Consistency (along with the assumptions of Theorem 2 ) is sufficient to pin down weights exactly. Theorem 3 details the claim:

Theorem 3 Consider $n$ preference relations, $\left\{\succeq_{i}: i=1,2, \ldots n\right\}$ defined on $\mathcal{G}$.Then the following two statements are equivalent:

1. Each $\succeq_{i}$ satisfies $A 1-A 6$.

2. For any two bargaining situations $f$ and $g, f \succ_{i} g$ if and only if

$$
\sum_{s \in \Pi_{i}} \frac{(n-s) !(s-1) !}{n !}[f(S)-f(S \backslash i)]>\sum_{s \in \Pi_{i}} \frac{(n-s) !(s-1) !}{n !}[g(S)-g(S \backslash i)]
$$

PROOF: See Appendix.

\section{Discussion}

This section elaborates on the issues raised in the last paragraph of the Introduction

There have been several formulations of the Shapley value for TU games besides Shapley's own seminal contribution. (See for e.g. Young(1988), Hart and Mas-Colell(1987), Chun(1989) and Roth(1977).) A typical characterization involves imposing a set of axioms on a cardinal function and showing that the Shapley value is the unique function that satisfies these axioms. The different axioms in these characterizations yield alternate interpretations to the value. Young(1988) is

\footnotetext{
${ }^{4}$ Consider the following axiom: $\mathrm{AXIOM}^{-}$5: (Anonymity) Let $S$ be a coalition that does not contain the three distinct players $i, j$ and $k$. For any real number $x$, if $x \delta_{s u i, j} \succ_{i} \delta_{i}$, then $x \delta_{s u i, k} \succ_{i} \delta_{i}$. Replacing the Symmetry axiom with the Anonymity axiom, one can show that, for each player, the weights obtained in Theorem 1 must depend only on the size of various coalitions. However, different players may yet have different sets of weights. If one imposes Anonymity along with the Symmetry, the weights coincide for all the players and depend only on the size of the various coalitions.
} 
motivated by ideas of distributive justice while Hart and Mas-Colell(1987) is motivated by notions of cost-allocation. However, the original motivation for the value (see Roth(1977)) is to regard it as the utility of a game.

To the best of my knowledge, Roth(1977) is the only characterization that does not impose efficiency as an axiom. Efficiency requires that the sum of the values of various players in a game add up to the worth of the grand coalition. If one were to interpret the value as the utility of a game, efficiency ${ }^{5}$ requires summing up the utilities of different players. It is then not clear as to what interpersonal comparisons of utility are being made ${ }^{6}$.

In the present context, Theorem 3 provides the utility of a game situation. Nowhere in my construction do I add the utilities or the representations of different players. In fact, axioms A1-A4 are restrictions on the preferences of a single player. It is only A5 and A6 that make a direct interpersonal comparison. While A5 is a symmetry assumption, A6 is a statement on the knowledge of the model among the players rather than a direct comparison of utility. Hence, Theorem 3 provides a proper basis for interpreting the value as the utility of a conflict situation.

Roth(1977) also describes a framework for obtaining the Shapley value without using efficiency as an axiom. Roth(1977) is concerned with the preferences of a single player who is uncertain about the TU game to be played and the position or the role that the player may be in. Allowing for lotteries over this space of uncertainty, he assumes that the player satisfies all the standard axioms of expected utility theory. Thus he obtains a von Neumann-Morgenstern(VNM) utility function, say $\theta$. If the realized outcome of the lottery is a game $v$ with the position $i$, then $\theta(v, i)$, is the VNM utility. Among others, neutrality to "strategic" and "ordinary" risk are shown to imply that $\theta(v, i)$ is in fact the Shapley value.

\footnotetext{
${ }^{5}$ Another important axiom used in most of the constructions is Additivity, that is the value of the sum of two different games add up to the sum of the values of the games. This assumption too is not meaningful in the present context.

${ }^{6}$ Nonetheless, some authors have associated the Shapley value with classical utilitarianism (See Moulin(1988)), as it seems to maximize the sum of utilities of the various players.
} 
The present work differs from Roth(1977) in two important ways. In the model presented in Section 2, there is no uncertainty about the games being played. Players are involved in a conceptual exercise of ranking one bargaining situation over another but never lotteries over bargaining situations. Consequently attitudes to risk are irrelevant. Second, since Roth(1977) obtains the Shapley value only as a VNM utility functional, the representation is unique only upto a positive linear transformation. On the other hand, the representation obtained in Theorem 3 is ordinal; it is unique upto a positive monotone transform.

Theorem 3 also determines a bargaining solution when side-payments are made in a single good. Players obtain a weighted average of their marginal contributions with the weights being the Shapley weights. There are two interesting features of this bargaining solution. First, we obtain a pareto-optimal allocation without resorting to efficiency as an axiom.

Second, for two player games, the Shapley value coincides with another widely studied sharing rule; the Nash Bargaining solution (See Nash(1950)). One of the axioms in the Nash bargaining solution requires that the physical outcome corresponding to the agreed utility payoff be invariant to positive linear transforms of the players' utilities over the underlying set of alternatives. This invariance of the physical outcome with respect to positive linear transforms is a natural requirement if one were bargaining over the exchange of goods by means of lotteries: decision-theoretically equivalent utility functions must yield the same physical outcome. However, in the present context where the players are bargaining over an infunitely divisible good, rather than lotteries over (perhaps indivisible) goods, one would naturally require (to prevent interpersonal comparisons of utility) the physical outcome be invariant to ordinal transforms of the players' utility for the underlying good.

However, it is shown in Shapley $(1969)^{7}$, that at least for two person games it is impossible to construct a theory where the physical outcome is invariant to ordinal transforms of utility. This is

\footnotetext{
${ }^{7}$ See also Chapter 5, Shubik(1982)
} 
sometimes referred to as the Bargainers" Paradox. In fact, this prompts Shapley(1969) to assert that:

Interpersonal comparability of utility ... enters naturally - and, I believe, properly - as a nonbasic, derivative concept playing an important if sometimes hidden role in theories of bargaining...

The problem pointed out in Shapley(1969) appears since axiomatic bargaining theory focuses on the shapes of the utility possibility frontiers and other such geometric criteria rather than the underlying physical or economic environment. This paper studies a restricted yet an interesting class of physical environments. The procedure adopted here yields a bargaining solution that is trivially immune to ordinal transforms of utility for the underlying good. This is perhaps the central contribution of the paper.

A by-product of this work is an NTU value when side-payments are possible only in a single good. A value for games with non- transferable utility(NTU) was formulated in Harsanyi(1963) followed by Shapley(1969). (See also Aumann(1985b) and Hart(1985).) The NTU value has been a subject of considerable controversy in the last decade or so. This controversy follows certain puzzling and counter-intuitive predictions the value offers for examples presented in Roth(1980). (See also Aumann(1986) and Roth(1987)). In particular, concern has also been expressed regarding two related issues: the interpersonal comparisons of utility implicit in the NTU value and how one might accommodate the various interpretations that are accorded to the value with transferable utility in the case of NTU. It is clear from the preceding discussion that the model presented here answers these concerns for the environments studied therein.

\section{Conclusion}

The primary motivation of the paper has been to see how a rational player evaluates certain conflict 
situations in the presence of other rational players. For the conflict situations considered here, the evaluation is a weighted average of his marginal contributions to the various coalitions. The interpersonal comparisons made are minimal and in our opinion, natural. Three central insights have been obtained by studying preference orderings over bargaining situations. First, we have a proper framework for interpreting the "Shapley value" as an ex-ante evaluation of a conflict situation. Second, an extension of the original interpretation of the value to the NTU case. And, most significantly, a bargaining solution invariant to ordinal transforms of utility for the underlying good.

The axioms laid out in Section 2 should be taken as primitive. However, various non-cooperative bargaining procedures do guide the intuition. While there are bargaining procedures ${ }^{8}$ that satisfy all our axioms ${ }^{9}$, it is easy to construct bargaining procedures that violate some or even all the axioms. Nonetheless, certain key axioms such as Consistency, Nullity and Productivity are satisfied by the equilibria of all the standard bargaining procedures.

Several extensions can be suggested. All the results in this paper hinge very critically on Strategic Equivalence. A weakening of this axiom would be of considerable interest. One suspects that any such weakening would lead to a loss of separability between the weighting measure and the bargaining situation.

Another important extension is to allow for side-payments in several goods. This extension seems rather complicated. This is because with more that one good it is not clear how the strategic position of a player changes when endowed with more of some of the goods.

\section{Appendix}

The set $\delta_{S}: S \subseteq N$ constitutes a basis for $\Re^{2^{N}-1}$. Hence every bargaining situation $f \in \mathcal{G}$, can be

\footnotetext{
${ }^{\circ}$ Of course, one has to specify the exact utility for the underlying good. The statements made here assume linearity. ${ }^{\ominus}$ See Hart and Mas-Colell(1992) and Gul(1985).
} 
expressed a unique linear combination of the elements of the above set. That is to say

$$
\begin{aligned}
f & =\sum_{S \subseteq N} \gamma_{f}(S) \delta_{S} \\
& =\sum_{\gamma_{f}(S) \geq 0} \gamma_{f}(S) \delta_{S}+\sum_{\gamma_{f}(S)<0} \gamma_{f}(S) \delta_{S} \\
& \equiv f^{+}-f^{-}
\end{aligned}
$$

Note that both $f^{+}$and $f^{-}$are both made up of non- negative linear combinations of the situations $\delta_{S}$. I will assume that $\succ_{i}$ satisfies Axioms 1-4, although some of them are superflous for certain lemmas.

Lemma 1 For any two bargaining situations $f$ and $g$,

1. Suppose $h \equiv h^{+}$. Then $f \succ_{i} g$ if and only if $f+h \succ_{i} g+h$.

2. $f \succ_{i} g$ if and only if $f^{+}+g^{-} \succ_{i} g^{+}+f^{-}$.

Proof: Part 1 of the Lemma follows from a repeated application of Strategic Equivalence. The proof of Part 2 is complete by taking $h=f^{-}+g^{-}$.

Now consider the extension $>_{i}^{*}$ of $\succ_{i}$ from $\mathcal{G}$ to $\Re^{2^{N}-1}$.

For any $f$ and $g$ in $\Re^{2^{N}-1}, f>_{i}^{*} g$ if and only if $f^{+}+g^{-} \succ_{i} g^{+}+f^{-}$.

By virtue of the fact that $\delta_{S}$ constitutes a basis for the space $\Re^{2^{N}-1},>_{i}^{*}$ is indeed an extension of $\succ_{i}$

Lemma 2 The relation $>_{i}^{*}$ is complete and for any three vectors $f, g$ and $h$ in $\Re^{2^{N}-1}, f>_{i}^{*} g$ if and only if $f+h>_{i}^{*} g+h$. 
Proof: Completeness of $>_{i}^{*}$ follows from completeness of $\succ_{i}$. To prove the latter part of the lemma, I first prove that for any two vectors $f, g \in \Re^{2^{N}-1}, f>_{i}^{*} g$ if and only if $f-g>_{i}^{*} 0$. Note that the following equation holds because both sides represent the same vector, $(f-g)$ :

$$
(f-g)^{+}-(f-g)^{-}=\left(f^{+}-f^{-}\right)-\left(g^{+}-g^{-}\right)
$$

or,

$$
(f-g)^{+}=\left(f^{+}+g^{-}\right)-\left(g^{+}+f^{-}\right)+(f-g)^{-}
$$

Now consider the following sequence obtained on using equation 1 :

$$
\begin{array}{lc} 
& f-g>_{i}^{*} 0 \\
\Leftrightarrow & (f-g)^{+} \succ_{i}(f-g)^{-} \\
\Leftrightarrow & \left(f^{+}+g^{-}\right)-\left(g^{+}+f^{-}\right)+(f-g)^{-} \succ_{i}(f-g)^{-}
\end{array}
$$

Adding $\left(g^{+}+f^{-}\right)$to both sides of the last relation , and using part 1 of Lemma 1 ,

$$
\begin{array}{lc} 
& (f-g)>_{i}^{*} 0 \\
\Leftrightarrow & \left(f^{+}+g^{-}\right)+(f-g)^{-} \succ_{i}\left(g^{+}+f^{-}\right)+(f-g)^{-} \\
\Leftrightarrow & \left(f^{+}+g^{-}\right) \succ_{i}\left(g^{+}+f^{-}\right) \\
\Leftrightarrow & f>_{i}^{*} g
\end{array}
$$

Now since $f$ and $g$ are arbitrary vectors, we have the following sequence:

$$
\begin{aligned}
f>_{i}^{*} g & \Leftrightarrow f-g>_{i}^{*} 0 \\
& \Leftrightarrow(f+h)-(g+h)>_{i}^{*} 0 \\
& \Leftrightarrow(f+h)>_{i}^{*}(g+h),
\end{aligned}
$$


REMARK: Lemma 2 shows that if $\succ_{i}$ satisfies Strategic Equivalence on $\mathcal{G}$, there is an extension $>_{i}^{*}$ to the entire space $\Re^{2^{N}-1}$ that satisfies an analogue Part 1 of Lemma 1 . Although the relation $>_{i}^{*}$ is complete, it need not be transitive. The following lemma, however isolates the property sufficient for our purposes.

Lemma 3 Suppose $f>_{i}^{*} g$ and $g>_{i}^{*} 0$. Then $f>_{i}^{*} 0$.

Proof: Suppose that, by way of contradiction, $0 \geq_{i}^{*} f$. Then using Lemma $1, f^{-}+g^{+} \succeq_{i} f^{+}+g^{+}$. Now, $g^{+} \succ_{i} g^{-}$by hypothesis. Hence using Lemma 1 , I get $g^{+}+f^{+} \succ_{i} g^{-}+f^{+}$. The previous two expressions and transitivity imply, $f^{-}+g^{+} \succ_{i} f^{+}+g^{-}$, or $g>_{f}^{*}$. This is not compatible with the hypothesis $f>_{i}^{*} g$. Hence, $f>_{i}^{*} g$. $\square$.

Lemma 4 Suppose $f \geq_{i}^{*} 0$. Then for any $\alpha \geq 0, \alpha f \geq_{i}^{*} 0$.

Proof: I will first prove the lemma when $\alpha$ is an integer. Suppose $f \geq_{i}^{*} 0$. Then $f^{+} \succeq_{i} f^{-}$. The proof is by induction. Suppose the lemma is true for any integer $\alpha \leq k$. Then $k f^{+} \succeq_{i} k f^{-}$. Now let $\alpha=k+1$. By Strategic Equivalence

$$
\begin{aligned}
& f^{+}+k f^{+} \succeq_{i} \quad f^{-}+k f^{+} \\
& k f^{+}+f^{-} \succeq_{i} \quad k f^{-}+f^{-}
\end{aligned}
$$

The above two equations together with transitivity yield $(k+1) f^{+} \succeq_{i}(k+1) f^{-}$.

I now claim that for any integer $k,\left(\frac{1}{k}\right) f^{+} \succeq_{i}\left(\frac{1}{k}\right) f^{-}$. Suppose not. Then $\left(\frac{1}{k}\right) f^{-} \succ_{i}\left(\frac{1}{k}\right) f^{+}$. But then multiplying both sides by $k, f^{-} \succ_{i} f^{+}$, a contradiction.

Using these two facts, it is easy to see that the lemma holds when $\alpha$ is a rational number. For a general $\alpha$, choose a sequence of rational numbers $\alpha_{n}$ such that $\lim _{n \rightarrow \infty} \alpha_{n}=\alpha$. Then $\alpha_{n} f^{+} \succeq_{i} \alpha_{n} f^{-}$. Since $\succeq_{i}$ is continuous, it follows that $\alpha f^{+} \succeq_{i} \alpha f^{-}$, or $\alpha f \geq_{i}^{*} 0$. This proves the lemma. 
Let $E_{i}$ denote the set of all $f$ that are strictly better than 0 , i.e.,

$$
E_{i}=\left\{f \in \Re^{2^{N}-1}: f>_{i}^{*} 0\right\}
$$

Lemma 5 The set $E_{i}$ is an open and convex subset of $\Re^{2^{N}-1}$.

Proof: I will first show that $E_{i}$ is open. Let $f_{*}$ be a limit point of $E_{i}^{c}$. Then there is an infinite sequence of points $f_{n}$ in $E_{i}^{c}$, such that $\lim _{n \rightarrow \infty} f_{n}=f_{*}$. For each $n, f_{n}^{-} \succeq_{i} f_{n}^{+}$. So the pair $\left(f_{n}^{-}, f_{n}+\right)$ is a point in the graph of $\succeq_{i}$. Since $f_{n}$ converges to $f_{n}$, it follows that $f_{n}^{+}$converges to $f_{*}^{+}$and $f_{n}^{-}$converges to $f_{*}^{-}$. By continuity, it follows that $\left(f_{*}^{-}, f_{*}^{+}\right)$is in the graph of $\succeq_{i}$. That is to say $f_{*}^{-} \succeq_{i} f_{*}^{+}$, or $f_{*} \in E_{i}^{c}$. Hence $E_{i}^{c}$ is closed, or $E_{i}$ is open.

Let $f, g \in E_{i}$. By virtue of Lemma 4 , for any $\alpha \in(0,1)$,

$$
\alpha f>_{i}^{*} 0 \quad \text { and } \quad(1-\alpha) g>^{*} 0
$$

Adding $\alpha f$ to both sides of the expression on the far right and using Lemma 2

$$
(1-\alpha) g+\alpha f>_{i}^{*} \alpha f
$$

Now using Lemma 3 I conclude that $(1-\alpha) g+\alpha f \in E_{i}$. Hence $E_{i}$ is convex. Q.E.D.

Lemma 6 There is a set of weights $\{\lambda(i, S): S \subseteq N\}$, such that for any $f, g \in \mathcal{G}$,

$$
f \succ_{i} g \Longleftrightarrow \sum_{S \subseteq N} \lambda(i, S) f(S)>\sum_{S \subseteq N} \lambda(i, S) g(S)
$$

Proof: By Lemma 5, the set $E_{i}$ is an open and convex subset of $\Re^{2^{N}-1}$ that does not contain the origin. Hence the separating hyperplane theorem applies. There is a hyperplane that strictly separates the origin and the set $E_{i}$. Let $\{\lambda(i, S): S \subseteq N\}$ denote this hyperplane. Hence $f \in E_{i}$ if and only if $\sum_{s \subseteq N} \lambda(i, S) f(S)>0$. 
Now consider any two bargaining situations $f$ and $g$ such that $f \succ_{i} g$. By virtue of Lemma 2, this is true if and only if $f-g>_{i}^{*} 0$, i.e. $f-g \in E_{i}$, or

$$
\sum_{S \subseteq N} \lambda(i, S)[f(S)-g(S)]>0
$$

This completes the proof. $\square$.

Lemma 7 Suppose that $S \in \Pi_{-i}$. Then $\lambda(i, S)+\lambda(i, S \cup i)=0$.

Proof: The proof is by induction on the size of the coalition. By Nullity, there is an $x \neq 1$ such that $\delta_{N \backslash i} \sim_{i} \alpha \delta_{N \backslash i}$. Using the representation in the previous lemma,

$$
\sum_{S} \lambda(i, S) \delta_{N \backslash i}=x \sum_{S} \lambda(i, S) \delta_{N \backslash i}
$$

Or,

$$
\lambda(i, N)+\lambda(i, N \backslash i)=0
$$

Now suppose that we have proved the lemma for every coalition $S \in \Pi_{-i}$ of size $k, N-1 \geq k \geq 2$. It remains to prove for the lemma for a coalition of size $k-1$. Let $S \in \Pi_{-i}$ be a coalition of size $k-1$. By Nullity, it follows that there is an $x \neq 1$ such that $\delta_{S} \sim_{i} x \delta_{S}$. Hence,

$$
\sum_{T \subset N} \lambda(i, T) \delta_{S}(T)=x \sum_{T \subset N} \lambda(i, T) \delta_{S}
$$

Or,

$$
\lambda(i, S)+\lambda(i, S \cup i)+\sum_{T \in \Pi_{-i}, T \supset S, t \geq k} \lambda(i, T)+\lambda(i, T \cup i)=0 .
$$

But by the induction hypothesis, second expression on the left hand side is zero. Hence,

$$
\lambda(i, S)+\lambda(i, S \cup i)=0
$$

This completes the proof. $\square$ 
Corollary 1 For any $S, T \in \Pi_{-i}$ and $x \geq 0, x \delta_{S} \sim_{i} \delta_{T}$.

PROOF: For any bargaining situation $f$, the following is true:

$$
\begin{aligned}
\sum_{S \subseteq N} \lambda(i, S) f(S)= & \sum_{s \in \Pi_{i}} \lambda(i, S) f(S)+\sum_{s \in \Phi_{i}} \lambda(i, S) f(S) \\
= & \sum_{s \in \Pi_{i}} \lambda(i, S) f(S)-\sum_{s \in \Pi_{-i}} \lambda(i, S \cup i) f(S)+ \\
& \sum_{s \in \Pi_{-i}} \lambda(i, S \cup i) f(S)+\sum_{s \in \Pi_{-i}} \lambda(i, S) f(S) \\
= & \sum_{S \in \Pi_{i}} \lambda(i, S)[f(S)-f(S \backslash i)]+ \\
& \sum_{S \in \Pi_{-i}}[\lambda(i, S)+\lambda(i, S \cup i)] f(S)
\end{aligned}
$$

By virtue of Lemma 7, it follows that the very last expression above is zero. Hence

$$
\sum_{S \in \Pi_{i}} \lambda(i, S) f(S)=\sum_{S \in \Pi_{i}} \lambda(i, S)[f(S)-f(S \backslash i)]
$$

The corollary is now an easy implication of the above expression.

Lemma 8 For every coalition $S \in \Pi_{i}, \lambda(i, S)>0$.

PROOF: We will first prove the statement when $S \neq i$. By virtue of Lemma 7 , it is sufficient to show that $\lambda(i, S \cup i)>\lambda(i, S)$ for each $S \in \Pi_{-i}$. Suppose, by way of contradiction, that $\lambda(i, S) \geq \lambda(i, S \cup i)$. Now by virtue of the previous lemma,

$$
\sum_{S \subset T, T \in \Pi_{-i}} \lambda(i, T)+\lambda(i, T \cup i)=0
$$

Hence,

$$
\lambda(i, S) \geq \lambda(i, S \cup i)
$$

Or,

$$
\lambda(i, S)+\sum_{S \subset T, T \in \Pi_{-i}}[\lambda(i, T)+\lambda(i, T \cup i)] \geq \lambda(i, S \cup i)+2 \sum_{S \subset T, T \in \Pi_{-i}}[\lambda(i, T)+\lambda(i, T \cup i)]
$$


Or,

$\lambda(i, S)+\lambda(i, S \cup i)+\sum_{S \subset T, T \in \Pi_{-i}}[\lambda(i, T)+\lambda(i, T \cup i)] \geq 2 \lambda(i, S \cup i)+2 \sum_{S \subset T, T \in \Pi_{-i}}[\lambda(i, T)+\lambda(i, T \cup i)]$ Or,

$$
\sum_{T \supseteq S} \lambda(i, T) \geq 2 \sum_{T \supseteq S \cup i} \lambda(i, T)
$$

But this is the same as saying,

$$
\delta_{S} \succeq_{i} 2 \delta_{S \cup i}
$$

By virtue of the previous corollary, the above is equivalent to

$$
\delta_{N \backslash S \cup i} \succeq_{i} 2 \delta_{S \cup i}
$$

This, contradicts Productivity.

We are now all set to prove Theorem 1.

PROOF OF THEOREM 1: It is easy to verify that 2 implies 1 . Now suppose 1 holds. I will show that 2 must hold as well. Lemma 6 and the expression obtained in the Corollary imply the following:

$$
f \succ_{i} g \Longleftrightarrow \sum_{S \in \Pi_{i}} \lambda(i, S)[f(S)-f(S \backslash i)]>\sum_{S \in \Pi_{i}} \lambda(i, S)[g(S)-g(S \backslash i)]
$$

By virtue of Lemma 8, each of the weights in the above expression is positive. Now we may obtain the weights statement of theorem 1 by defining $p(i, S)$ as follows:

$$
p(i, S)=\frac{\lambda(i, S)}{\sum_{s \in \Pi_{i}} \lambda(i, S)}
$$

The set of weights $p(i)=\left\{p(i, S): S \in \Pi_{i}\right\}$ is said to represent $\succ_{i}$. It remains to demonstrate the uniqueness of these weights. To do this, it is useful to introduce some notation. Given a set of 
weights $p(i)$, such the $p(i, S)>0$ and $\sum_{S \in \Pi_{i}} p(i, S)=1$, define:

$$
\alpha_{p(i)}(S)=\sum_{T \in \Pi_{i}} p(i, T)\left[\delta_{S}(T)-\delta_{S}(T \backslash i)\right.
$$

For any $S \in \Pi_{i}, \alpha_{p(i)}(S)$ denotes the evaluation of the situation $\delta_{S}$ by player $i$ under the weights $p(i)$. Note that $\alpha_{p(i)}(i)=1$ and $\alpha_{p(i)}(S)>0$. Now label the sets $S \in \Pi_{i}$ and fix the order. Let $\alpha_{p(i)}$ denote the vector in $\Re^{2^{N-1}}$ whose entries correspond to $\alpha_{p(i)}(S)$ and the order is as per the earlier labelling of the coalitions. Assume without loss of generality that $\alpha_{p(i)}(i)$ is the first coordinate.

Now for each $S \in \Pi_{i}$, let $\delta_{S}^{i}$ be the vector in $\Re^{2^{N-1}}$ obtained from each $\delta_{S} \in \Pi_{i}$ by restricting to the coalitions that contain player ${ }^{10} i$. Let $\mathbf{M}_{i}$ denote the matrix ${ }^{11}$ whose rows are composed of the vectors $\delta^{i}(S)$. The $k^{\text {th }}$ row corresponds to the $k^{\text {th }}$ set $S \in \Pi_{i}$ as per the earlier labelling.

Then, by construction,

$$
\mathbf{M}_{i} p(i)=\alpha_{p(i)}
$$

Now let if possible $q(i)$ be another set of positive weights that sum to one and represent $\succ_{i}$. It is easy to see that $\mathbf{M}_{i}$ has full rank. Hence, $p(i) \neq q(i)$, implies that there is a coalition $S$ such that $\alpha_{p(i)}(S) \neq \alpha_{q(i)}(S)$. Assume, without loss of generality, that $\alpha_{p(i)}(S)>\alpha_{q(i)}(S)$. Now consider the situation $f^{*}$ defined as follows:

$$
f^{*}=\frac{1}{\sqrt{\alpha_{p(i)}(S) \alpha_{q(i)}(S)}} \delta_{S}
$$

Now, using $p(i)$,

$$
\sum_{S \in \Pi_{i}} p(i, S)\left[f^{*}(S)-f^{*}(S \backslash i)\right]=\sqrt{\frac{\alpha_{p(i)}(S)}{\alpha_{q(i)}(S)}}>1
$$

Or,

$$
f^{*} \succ_{i} \delta_{i}
$$

\footnotetext{
${ }^{10}$ For e.g. with two players $A$ and $B,\left(\delta_{A}(A), \delta_{A}(B), \delta_{A}(A B)\right)=(1,0,1)$ and $\left(\delta_{A}^{A}(A), \delta_{A}^{A}(A B)\right)=(1,1)$; $\left(\delta_{A B}(A), \delta_{A B}(B), \delta_{A B}(A B)\right)=(0,0,1)$ and $\left(\delta_{A B}^{A}(A), \delta_{A B}^{A}(A)\right)=(0,1)$.

${ }^{11}$ For the two player case, $M_{A}=\left(\begin{array}{c}\delta_{A}^{A} \\ \delta_{A B}^{A}\end{array}\right)=\left(\begin{array}{ll}1 & 1 \\ 0 & 1\end{array}\right)$
} 
Now using $q(i)$ instead of $p(i)$, we obtain

$$
\delta_{i} \succ_{i} f^{*}
$$

which is a contradiction. It is now easy to see that ${ }^{12} \alpha_{p(i)}=\alpha_{q(i)}$. This concludes the proof.

PROOF OF THEOREM 2: Let $i, j \in S$. We will first show that $\alpha_{p(i)}(S)=\alpha_{p(j)}(S)$. Assume, by way of contradiction, that $\alpha_{p(i)}(S)>\alpha_{p(j)}(S)$. Set $\alpha=\frac{1}{\sqrt{\alpha_{p(i)}(S) \alpha_{p(j)}(S)}}$. Now,

$$
\sum_{T \in \Pi_{i}} p(i, S)\left[\delta_{S}(T)-\delta_{S}(T \backslash i)\right]=\sqrt{\frac{\alpha_{p(i)}(S)}{\alpha_{q(i)}(S)}}>1
$$

Hence $\alpha \delta_{s} \succ_{i} \delta_{i}$. A similar exercise as above yields $\delta_{j} \succ_{j} \alpha \delta_{s}$. This violates Symmetry.

Hence $\alpha_{p(i)}(S) \leq \alpha_{q(i)}(S)$. Since a strict inequality yields a contradiction as the one above, it follows that

$$
\alpha_{p(i)}(S)=\alpha_{p(j)}(S)
$$

To show that $p(i, S)=p(j, S)$, first note that when $S=N$,

$$
p(i, N)=\alpha_{p(i)}(N)=\alpha_{p(j)}(N)=p(j, N)
$$

Now suppose that we have proven $p(i, S)=p(j, S)$ for every coalition $S$ of size $k+1,1 \leq k \leq N-1$. If we show that the equality must hold for a coaction of size $k$, then the proof is complete by induction on the size of the coalitions. Let $S$ be a coalition of size $k$. Then by the induction hypothesis,

$$
\sum_{T \supset S} p(i, T)\left[\delta_{S}(T)-\delta_{S}(T \backslash i)=\sum_{T \supset S} p(j, T)\left[\delta_{S}(T)-\delta_{S}(T \backslash j)\right.\right.
$$

Assume by way of contradiction that $p(i, S)>p(j, S)$. Then,

$$
\sum_{T \supseteq S} p(i, T)\left[\delta_{S}(T)-\delta_{S}(T \backslash i)>\sum_{T \supseteq S} p(j, T)\left[\delta_{S}(T)-\delta_{S}(T \backslash j)\right.\right.
$$

\footnotetext{
${ }^{12}$ Note that we have critically used the fact that the set of weights sum up to one, or $\alpha_{p(i)}(i)=\alpha_{q(i)}(i)=1$.
} 
Or, $\alpha_{p(i)}(S)>\alpha_{p(j)}(S)$, a contradiction. Hence, $p(i, S) \leq p(j, S)$. Since a strict inequality yields a similar contradiction, if follows that $p(i, S)=p(j, S)$.

PROOF OF THEOREM 3: From Theorem 2, it is clear that for every coalition $S$, there is a unique number $\alpha(S)$, such that $i \in S, \alpha(S) \delta_{S} \sim \delta_{i}$. I now claim that Consistency implies that $\alpha(S)=|S|$

Suppose that, by way of contradiction, that $\alpha(S)>|S|$. Hence,

$$
\delta_{i} \sim \alpha(S) \delta_{S} \succ_{i}|S| \delta_{S} \quad \text { for all } i \in S,
$$

for some $\alpha>|S|$. But clearly, this implies all the players in $S$ are pessimists. This violates Part 2 of Consistency. Hence $\alpha(S) \leq|S|$. Since a strict inequality leads to a contradiction of Part 1 of the Consistency, one concludes that $\alpha(S)=|S|$. Hence $|S| \delta_{S} \sim_{i} \delta_{i}$, or

$$
|S| \sum_{T \in \Pi_{i}} p(i, T)\left[\delta_{S}(T)-\delta_{S}(T \backslash i)\right]=\sum_{T \in \Pi_{i}} p(i, T)\left[\delta_{i}(T)-\delta_{i}(T)\right]
$$

Or,

$$
\sum_{T \in \Pi_{i}} p(i, S) \delta_{S}(T)=\frac{1}{|S|} \quad S \in \Pi_{i}
$$

The Shapley weights solve the above system of equations. Since the solution must be unique, it follows that:

$$
p(i, S)=\frac{(n-s) !(s-1) !}{n !}
$$

This completes the proof. $\square$. 


\section{References}

AUMANN, R.J. (1985a): On the Non-Transferable Utility Value: A comment on the Roth Shafer Examples, Econometrica, 54, 667-77.

AUMANN, R.J. (1985b): An Axiomatization of the Non- Transferable Utility Value, Econometrica, 53, 599-612.

AUMANN, R.J. (1986): Rejoinder, Econometrica, 54, 985- 989.

CHUN, Y. (1989): "A New Axiomatization of the Shapley Value," Games and Economic Behaviour, vol 1, no:2, June, 119-130. GUL, F. (1985): "Bargaining foundations of the Shapley Value," Econometrica, 57, 81-95.

HART, S. (1985): An Axiomatization of Harsanyi"s Non- transferable utility Solutions, Econometrica, 53, 1295-1313.

HART, S. and Mas-COLELL, A. (1992): "A Model of n- Person Non- Cooperative Bargaining", Working Paper Series, Centre for Rationality and Interactive Decision Theory, Jerusalem, ISRAEL. HART, S. and Mas-COLELL, A. (1987): "Potential, Value and Consistency," Econometrica, 57, 589-614.

MOULIN, H. (1988): Axioms of Cooperative Decision Making, New York: Cambridge University Press.

NASH, J. (1950): " The Bargaining Problem," Econometrica, 28, 155-162.

ROTH A.E. (1977): "The Shapley Value as a von Neumann- Morgenstern Utility," Econometrica, $45,657-664$.

ROTH A.E. (1980): "Values for games without Side- Payments: Some Difficulties with Current Concepts," Econometrica, 48, 457-65.

ROTH A.E. (1986): "On the Non-Transferable Utility Value: A Reply to Aumann," Econometrica, 981-984. 
ROTH A.E. (1988): The Shapley Value, New York: Cambridge University Press.

SHAPLEY, L.S (1953): "A value for n-Person Games," reprinted in The Shapley Value, Alvin Roth ed., New York: Cambridge University Press.

SHAPLEY, L.S. (1969): "Utility Comparison and the theory of games," reprinted in The Shapley Value, Alvin Roth ed., New York: Cambridge University Press.

YOUNG H.P. (1988): "Individual Contribution and Just Compensation," in The Shapley Value, Alvin Roth ed., New York: Cambridge University Press. 